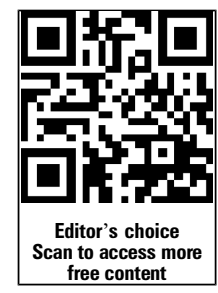

SHORT REPORT

\title{
Contribution of sexual practices (other than anal sex) to bacterial sexually transmitted infection transmission in men who have sex with men: a cross-sectional analysis using electronic health records
}

\author{
Jessica L Nash, 1,2,3 Jane S Hocking, ${ }^{1,2}$ Tim R H Read, 1,2 Marcus Y Chen, 1,2 \\ Catriona S Bradshaw, ${ }^{1,2,3}$ Dana S Forcey, ${ }^{1,2}$ Christopher K Fairley ${ }^{1,2}$
}

${ }^{1}$ Melbourne Sexual Health Centre, Melbourne, Victoria, Australia

${ }^{2}$ Sexual Health Unit, Melbourne School of Population and Global Health, University of Melbourne, Melbourne, Victoria, Australia ${ }^{3}$ Department of Epidemiology and Preventive Medicine, Monash University, Victoria, Australia

\section{Correspondence to} Dr Christopher K Fairley, University of Melbourne \& Director, Melbourne Sexual Health Centre, 580 Swanston Street, Carlton, Victoria 3053, Australia;

cfairley@mshc.org.au

Received 21 February 2013 Revised 20 June 2013 Accepted 2 September 2013 Published Online First 26 September 2013
To cite: Nash JL, Hocking JS, Read TRH, et al. Sex Transm Infect 2014;90:55-57.

\section{ABSTRACT}

Objective We quantified the proportion of cases and risk of primary syphilis (PS), urethral chlamydia (UCT) and urethral gonorrhoea (UGC) attributable to sexual practices other than anal sex.

Methods In this cross-sectional study, electronic records for men who have sex with men (MSM) who attended the Melbourne Sexual Health Centre between July 2002 (for PS) or January 2006 (for UCT and UGC) and October 2012, inclusive, were examined.

Results There were 37533 eligible consultations; 2374 $(6 \%)$ of these reported no anal sex. There were 204 PS diagnoses, 673 UCT diagnoses, and 618 UGC diagnoses; 12 (6\%), $16(2 \%)$ and 44 (7\%) cases, respectively, occurred in consultations where no anal sex was reported in the previous 3 months (PS, UGC) or twelve months (UCT). Among MSM reporting no anal sex, PS was diagnosed in 0.5 cases $/ 100$ consultations, UCT was diagnosed in 1.5 cases/100 tests for UCT and UGC was diagnosed in 14 cases/100 tests for UGC. UCT was significantly more common in MSM reporting anal sex (OR 2.18, 95\% Cl 1.32 to 3.59, $p=0.002)$, but PS (OR $1.0795 \% \mathrm{Cl} 0.6$ to $1.93, \mathrm{p}=0.82$ ) and UGC (OR $1.2895 \% \mathrm{Cl} 0.92$ to 1.79. $p=0.14$ ) were not. For MSM reporting anal sex, condom use was protective for all three infections (all $p \leq 0.03$ ).

Conclusions Our findings suggest that UCT uncommonly occurs from sexual practices other than anal sex; however, these practices contribute significantly to PS and UGC. Successful programmes to control PS and UGC will need strategies, such as frequent testing, in addition to promoting condom use.

\section{BACKGROUND}

Rates of syphilis, chlamydia and gonorrhoea among men who have sex with men (MSM) are continuing to rise. ${ }^{1}$ Subsequently, a number of public health strategies have been implemented to promote condom use for anal sex among MSM. ${ }^{2}$ However, MSM also take part in other sexual practices, including oral sex, nudging, dipping and rimming, for which condoms are either not suitable or not used. ${ }^{3}{ }^{4}$ In particular, oral sex is much more commonly practiced than anal sex among MSM. ${ }^{5}$

If these practices play a significant role in the transmission of sexually transmitted infections
(STI), then STI rates may continue to increase, despite high rates of condom use for anal sex. Our objective was to quantify the proportion of cases and risk of primary syphilis (PS), urethral chlamydia (UCT) and urethral gonorrhoea (UGC) attributable to sexual practices other than anal sex.

\section{METHODS}

\section{Study setting}

This study was conducted at Melbourne Sexual Health Centre. Data was extracted from electronic medical records, including self-reported behavioural information, and a diagnosis code. All records for male patients who attended between 1 July 2002 (for PS) or 1 January 2006 (for UCT and UGC-when laboratory results were computerised) and 31 October 2012, were included.

A swab of the urethra or discharge was tested for urethral Neisseria gonorrhoeae from men with significant urethral symptoms, and plated on Thayer-Martin medium. Urethral swabs, or firstpass urine, were used to test for urethral Chlamydia trachomatis (BD ProbeTec Strand Displacement Amplification). Syphilis staging was completed by a senior sexual health clinician, following clinical assessment and investigation (dark-ground microscopy, Treponema pallidum PCR, and serological tests (enzyme immunoassay, $T$ pallidum particle agglutination and rapid plasmid reagin)).

Men were included in the study if they reported at least one male sexual partner in the preceding 12 months and excluded if they were sex workers.

Data analysis

PS and UGC analyses were conducted for all MSM-including those with female partners-as these infections are rare in heterosexuals in Victoria. The UCT analysis was restricted to MSM who only had male partners, as chlamydia occurs commonly in heterosexual men.

SPSS v21 was used to generate crude and adjusted ORs and 95\% CIs. Associations were tested using the $\chi^{2}$ test, and generalised estimating equations were used to adjust for repeated measures from the same individual.

The Alfred Human Research Ethics Committee provided approval (525/12). 


\section{RESULTS}

There were 37553 eligible consultations (2002-2012); 2374 (6\%) reported no anal sex.

\section{Primary syphilis}

Totally, 204 cases of PS were diagnosed; of these, 12 cases $(6 \%$, 0.5 cases/ 100 consultations) were of men who reported no anal sex, 51 cases $(25 \%, 0.4$ cases/100 consultations) in men who reported anal sex with condoms 'always', and 132 cases $(65 \%$, 0.6 cases $/ 100$ consultations) in men who reported anal sex with condoms 'less than always'. The crude OR for PS in those who reported any anal sex was 1.07 (95\% CI 0.6 to 1.93 ) versus no anal sex.

After adjustment, men who reported anal sex were not at increased risk of PS as compared with men who reported no anal sex $(\mathrm{OR}=0.79,95 \% \mathrm{CI} 0.42$ to $1.49, \mathrm{p}=0.5$ for 'always used' condoms, and $\mathrm{OR}=1.24,95 \%$ CI 0.69 to $2.24, \mathrm{p}=0.44$ for used condoms 'less than always') (table 1).

\section{Urethral chlamydia}

Totally, 673 cases of UCT were diagnosed; of these, 16 cases $(2.4 \%, 1.5$ cases/100 tests) were of men who reported no insertive anal sex, 194 cases (29\%, 2.4 cases/100 tests) in men who reported insertive anal sex with condoms 'always', and 455 cases $(68 \%, 3.8$ cases/100 tests) in men who reported insertive anal sex with condoms 'less than always'. The crude OR for UCT in those who reported any insertive anal sex was 2.18 (95\% CI 1.32 to 3.59) versus no anal sex.

After adjustment, men who reported insertive anal sex were at increased risk of UCT as compared with men who reported no insertive anal sex $(\mathrm{OR}=1.72,95 \%$ CI 1.03 to $2.88, \mathrm{p}=0.04$ for 'always used' condoms, and $\mathrm{OR}=2.76,95 \%$ CI 1.67 to 4.55, $\mathrm{p}<0.001$ for used condoms 'less than always') (table 1).

\section{Urethral gonorrhoea}

Totally, 618 cases of UGC were diagnosed; of these, 44 cases (7.1\%, 14 cases/100 tests) were in men who reported no insertive anal sex, 169 cases (27\%, 15.1 cases/100 tests) in men who reported insertive anal sex with condoms 'always', and 402 cases $(65 \%, 18.4$ cases/100 tests) in men who reported insertive anal sex with condoms 'less than always'. The crude OR for UGC in those who reported any insertive anal sex was 1.28 (95\% CI 0.92 to 1.79 ) versus no anal sex.

After adjustment, men who reported insertive anal sex were not at increased risk of UGC as compared with men who reported no insertive anal sex $(\mathrm{OR}=0.98,95 \% \mathrm{CI} 0.68$ to 1.42 , $\mathrm{p}=0.92$ for 'always used' condoms, and $\mathrm{OR}=1.39,95 \% \mathrm{CI}$ 0.97 to $1.98, p=0.07$ for used condoms 'less than always') (table 1).

\section{DISCUSSION}

We found a substantial and significant risk of PS and UGC for men who had not had anal sex, and this risk was not different to the risk of these infections in men reporting anal sex. By contrast, the risk of UCT was significantly higher for men reporting anal sex.

Our study is the largest investigating the infection risk conferred by sexual practices, has findings which are more precise than those previously published, and is one of only four to incorporate a multivariate analysis. ${ }^{4-8}$ The largest and most

Table 1 Frequencies and multivariate analysis for cases of primary syphilis, urethral chlamydia, and urethral gonorrhoea

\begin{tabular}{|c|c|c|c|c|c|c|c|c|c|}
\hline & \multicolumn{3}{|l|}{ Primary syphilis } & \multicolumn{3}{|c|}{ Urethral chlamydia } & \multicolumn{3}{|c|}{ Urethral gonorrhoea } \\
\hline & Frequency (\%)* & Adjusted OR & p Value & Frequency (\%)† & Adjusted OR & p Value & Frequency (\%)‡ & Adjusted OR & p Value \\
\hline \multicolumn{10}{|l|}{ Age } \\
\hline$<35$ years & $110(0.5)$ & 1 & & $424(3)$ & 1 & & 409 (19.2) & 1 & \\
\hline$>35$ years & $94(0.7)$ & $1.35(1-1.81)$ & 0.05 & $249(3.3)$ & 1.02 & 0.78 & $209(13.5)$ & 0.62 & $<0.001$ \\
\hline \multicolumn{10}{|c|}{ Sexual practices over last 3 months§ } \\
\hline 0-1 male partners & $57(0.5)$ & 1 & & - & - & - & $111(12.8)$ & 1 & \\
\hline 2-4 male partners & $71(0.5)$ & $1.09(0.75-1.59)$ & 0.65 & - & - & - & $261(17)$ & $1.39(1.07-1.8)$ & 0.01 \\
\hline $5+$ male partners & $73(0.6)$ & $1.24(0.85-1.81)$ & 0.27 & - & - & - & $239(19.3)$ & $1.63(1.26-2.12)$ & $<0.001$ \\
\hline No IAS, no RAS & $12(0.5)$ & 1 & & - & - & - & - & - & - \\
\hline IAS and/or RAS, CA & $51(0.4)$ & $0.80(0.43-1.51)$ & 0.5 & - & - & - & - & - & - \\
\hline IAS and/or RAS, $C<A$ & $132(0.6)$ & $1.26(0.7-2.28)$ & 0.44 & - & - & - & - & - & - \\
\hline No IAS & - & - & - & - & - & - & $44(14)$ & 1 & \\
\hline IAS, CA & - & - & - & - & - & - & $169(15.1)$ & $0.98(0.68-1.42)$ & 0.92 \\
\hline IAS, $C<A$ & - & - & - & - & - & - & $402(18.4)$ & $1.39(0.97-1.98)$ & 0.07 \\
\hline \multicolumn{10}{|c|}{ Sexual practices over the last 12 months§ } \\
\hline$<5$ male partners & - & - & - & $195(2.6)$ & 1 & & - & - & - \\
\hline 5-10 male partners & - & - & - & $222(3.2)$ & $1.24(1.01-1.52)$ & 0.04 & - & - & - \\
\hline $10+$ male partners & - & - & - & $256(3.6)$ & 1.37 (1.13-1.67) & 0.002 & - & - & - \\
\hline No IAS & - & - & - & $16(1.5)$ & 1 & & - & - & - \\
\hline IAS, CA & - & - & - & $194(2.4)$ & $1.72(1.03-2.88)$ & 0.04 & - & - & - \\
\hline IAS, $\mathrm{C}<\mathrm{A}$ & - & - & - & $455(3.8)$ & $2.76(1.67-4.55)$ & $<0.001$ & - & - & - \\
\hline
\end{tabular}

*For all MSM with at least one male partner in the previous 12 months. Both IAS and RAS were taken into account during analysis. OR adjusted for age and number of male partners. tFor MSM who only had male partners in the previous 12 months, tested for chlamydia, from 2006 onwards. IAS only taken into account during analysis (as infections at non-urethral sites irrelevant). OR adjusted for age, number of male partners, and symptoms at triage.

¥For all MSM with at least one male partner in the previous 12 months, tested for gonorrhoea, from 2006 onwards. IAS only taken into account during analysis (as infections at non-urethral sites irrelevant). OR adjusted for age, number of male partners, and symptoms at triage.

$\S$ For PS and UGC analyses, sexual practices over the last 3 months were analysed. For UCT, sexual practices over the last 12 months were analysed, as it is thought to have a longer incubation period.

$C<A$, condoms used 'less than always'; $C A$, condoms 'always used'; IAS, insertive anal sex; RAS, receptive anal sex. 
recent study, with 5715 consultations, found that MSM who reported only oral sex in the preceding three months accounted for $5.1 \%$ of chlamydia cases, and $6.5 \%$ of gonorrhoea cases ${ }^{4}$; proportions comparable to our findings. Other smaller studies found associations between oral sex and gonorrhoea ${ }^{6}$ and chlamydia. $^{8}$

We did not have detailed information on specific non-anal sex sexual practices. These practices, for which condoms are rarely used, are very likely to have included oral sex, ${ }^{5}$ as well as dipping and nudging. ${ }^{3}$ If non-anal sex sexual practices (that still involve the anus) such as nudging or dipping explained a significant proportion of infections, then one might expect our findings to be similar for each of the three infections we studied. ${ }^{3}$ However, this was not the case; the risk associated with 'no anal sex' for each infection was different, suggesting that other practices (possibly oral sex) were the key factor that differentiated between UCT and the other two infections. This explanation is supported by prevalence data, which shows pharyngeal chlamydia is much less common than pharyngeal gonorrhoea. ${ }^{9}$

Our study, like other similar studies, used self-reported data, which is subject to social desirability and recall bias, particularly in relation to the frequency of condom use. ${ }^{10}$ However, we found that condom use was protective among those who reported anal sex for all three infections, which suggests this information was mostly accurate. The different findings between the three infections also suggest that these are real differences, which are not due to systematic bias.

Our results suggest that having anal sex does not increase the risk of syphilis or gonorrhoea transmission by more than twofold (upper CI). However, it would not be correct to conclude that anal sex does not carry additional risk. One possible explanation may be that non-anal sex sexual practices are different (and possibly more likely to transmit infection) in men who don't have anal sex. This might be the case if, for example, the non-anal sex sexual practices (such as oral sex) lasted longer when the sexual interaction did not involve anal sex. Unfortunately, we did not have detailed information on non-anal sex sexual practices, and there are no published studies exploring these practices sufficiently to answer this question.

Our studies, and other similar studies, reported sexual practices over a period of time (usually 3 months) and not per partner. Therefore, when someone reported having 10 sexual partners, and having anal sex with condoms always, it does not mean that anal sex had taken place with all partners, only that it had occurred with between one and 10. By contrast, oral sex is very common, and so it is likely that it occurred in almost all encounters. Thus, discerning the risk conferred by specific practices from data from studies similar to ours or others', is difficult. It is also unlikely that data with sufficient detail and statistical power could be collected without a very large and expensive study.

These findings have implications. Firstly, it is important that the MSM are aware that sexual practices other than anal sex pose a risk of infection, although this should be phrased in a way that does not discourage condom use for anal sex. It is also important to continue testing of the pharynx for gonorrhoea in
MSM, with the most sensitive methods (ie, nucleic acid amplification), so that asymptomatic infections are identified and treated and onward transmission terminated. Finally, public health strategies-while continuing to promote condom usealso need to highlight the importance of shortening the duration of infection through frequent testing if reductions in STIs are to be achieved in MSM.

\section{Key messages}

Sexual practices, other than anal sex, uncommonly contribute to urethral chlamydia transmission among MSM.

- However, these practices contribute significantly to cases of syphilis and urethral gonorrhoea.

- To successfully control these infections, programmes will need to use strategies, such as frequent testing, in addition to promoting condom use.

Handling editor Jackie A Cassell

Acknowledgements We thank Jun Kit Sze and Afrizal for their assistance with data extraction, and lan Denham for the coding of syphilis cases.

Contributors CKF and MYC conceived the study. JLN and CKF wrote the manuscript and analysed the data. JSH assisted with the data analysis. All authors provided intellectual input into the design and manuscript.

Competing interests None.

Ethics approval Alfred Health, Human Research Ethics Committee.

Provenance and peer review Not commissioned; externally peer reviewed.

\section{REFERENCES}

1 The Kirby Institute. Annual Surveillance Report 2012: HIV, viral hepatitis and sexually transmissible infections in Australia. In: McDonald A, ed. Annual Surveillance Report 2012. Sydney: NSW The University of New South Wales, 2012.

2 Department of Health and Ageing. Second National Sexually Transmissible Infections Strategy 2010-2013 Australian Government, 2010.

3 Phang CW, Hocking J, Fairley CK, et al. More than just anal sex: the potential for sexually transmitted infection transmission among men visiting sex-on-premises venues. Sex Transm Infect 2008;84:217-19.

4 Bernstein KT, Stephens SC, Barry PM, et al. Chlamydia trachomatis and Neisseria gonorrhoeae Transmission from the Oropharynx to the Urethra among Men Who Have Sex with Men. Clin Infect Dis 2009;49:1793-97.

5 Grulich AE, de Visser RO, Smith AMA, et al. Homosexual experience and recent homosexual encounters Australian and New Zealand. J Public Health 2003;27:155-63.

6 Lafferty WE, Hughes JP, Handsfield HH. Sexually Transmitted Diseases in Men Who Have Sex With Men. Sex Transm Dis 1997;24:272-78.

7 Benn PD, Rooney G, Carder C, et al. Chlamydia trachomatis and Neisseria gonorrhoeae infection and the sexual behaviour of men who have sex with men. Sex Transm Infect 2007:83:106-12.

8 Jin F, Prestage GP, Mao L, et al. Incidence and risk factors for urethral and anal gonorrhoea and chlamydia in a cohort of HIV-negative homosexual men: the Health in Men Study. Sex Transm Infect 2007;83:9.

9 Kent CK, Chaw JK, Wong W, et al. Prevalence of Rectal, Urethral, and Pharyngeal Chlamydia and Gonorrhea Detected in 2 Clinical Settings among Men Who Have Sex with Men: San Francisco, California, 2003. Clin Infect Dis 2005;41:67-74.

10 Vodstrcil LA, Fairley CK, Chen MY, et al. Risk-based HIV testing of men who have sex with men would result in missed HIV diagnoses. Sex Transm Dis 2012;39:492. 